Chirurg 2012 $\cdot 83: 994$

DOI 10.1007/s00104-012-2380-0

Online publiziert: 24. Oktober 2012

๑) Springer-Verlag Berlin Heidelberg 2012

O. Strobel · M.W. Büchler

Klinik für Allgemein-, Viszeral- und Transplantationschirurgie, Universität Heidelberg

\title{
Intermittierendes Pringle-Manöver: keine Reduktion des Blutverlustes in aktueller RCT
}

Gruppe und 335 (40-3160) $\mathrm{ml}$ in der KPM-Gruppe ( $p=1,000)$. Auch bezüglich des Blutverlustes in den verschiedenen Operationsphasen (vor Resektion, Resektionsphase, nach Resektion), des Blutverlustes pro Resektionsfläche, der Transfusionsrate und hinsichtlich der Operationsdauer ergaben sich keine Unterschiede zwischen den Gruppen. Allerdings waren die postoperativen ALT (Alanin-AminoTransferase)-Spiegel und die Morbidität (IPM: 41\% vs. KPM: 24\%) in der IPMGruppe signifikant erhöht.

\section{Diskussion und Fazit}

Die Studie zeigt, dass die routinemäßige Anwendung eines IPM nicht zu einer Reduktion des Blutverlustes führt, jedoch mit erhöhten Transaminasewerten als Zeichen eines potenziellen Ischämiereperfusionsschadens und möglicherweise auch mit einer erhöhten Morbidität verbunden ist.

Der Verlust des in früheren Studien beschriebenen Vorteils des IPM hinsichtlich einer Reduktion des Blutverlustes ist durch die zwischenzeitliche deutliche Reduktion des Blutverlustes auch ohne Pringle-Manöver von früher 2 I auf aktuell $300-400 \mathrm{ml}$ zu erklären und spiegelt Fortschritte in der Leberchirurgie wie verbesserte Transektionstechniken wider. Der Nutzen eines IPM hängt vom zu erwartenden Blutverlust $a b$, der wiederum von Faktoren wie Resektionsausmaß, Parenchymbeschaffenheit, Transektionstechnik und zu erreichendem ZVD (möglichst $<5 \mathrm{mmHG}$ ) bestimmt wird. Während eine routinemäßige Anwendung des IPM bei Leberresektionen an- hand der aktuellen Datenlage nicht zu empfehlen ist, kann ein Pringle-Manöver bei ausgewählten Patienten mit erwartet hohem Blutverlust oder vorbestehender Anämie sinnvoll sein.

\section{Korrespondenzadresse}

PD Dr. O. Strobel

Klinik für Allgemein-, Viszeralund Transplantationschirurgie, Universität Heidelberg, Im Neuenheimer Feld 110, 69120 Heidelberg Oliver.Strobel@med.uni-heidelberg.de

Interessenkonflikt. Der korrespondierende Autor gibt für sich und seinen Koautor an, dass kein Interessenkonflikt besteht. 